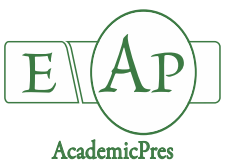

Radutoiu D and Cosmulescu S (2020)

Notulae Botanicae Horti Agrobotanici Cluj-Napoca 48(3):1709-1718

DOI: $10.15835 /$ nbha48311996

Note

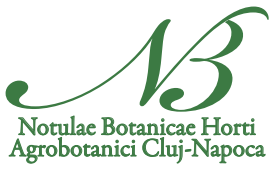

\title{
Chorological data regarding the presence of Corylus colurna species in Romania
}

\section{Daniel RĂDUȚOIU, Sina COSMULESCU*}

\author{
University of Craiova, Horticulture Faculty, A.I. Cuza Street, 13, Craiova, \\ Romania; sinacosmulescu@hotmail.com (*corresponding author); radutoiuO2daniel@gmail.com
}

\begin{abstract}
The Turkish hazel or Turkish filbert (Corylus colurna) is a less widespread tree species in Romania. Data on the presence of $C$. colurna species in the Romanian flora are quite rare. The article's aim is to analyse the scientific information and those of the main herbariums regarding the presence of $C$. colurna species on the Romanian territory. The presence of the species on the Romanian territory was signalled about 122 years ago in the paper "Conspectus of Romanian Flora". Subsequent scientific information identifies the species in different locations, especially in the southwest of Romania, in dendrological and natural parks. Scientific importance of the species lies in its rarity, the species has an area limit on Romanian territory and in the value, it gives to natural ecosystems in which it grows. The practical value is given by its edible fruits, valuable from a food or medicinal point of view and by its agronomic characteristics. As a result, the biodiversity conservation strategy should include forest protected areas as important objectives.
\end{abstract}

Keywords: chorological data; Corylus colurna; distribution; plants; Romania

\section{Introduction}

Corylus genus belongs to Corylaceae family (Dihoru, 1975; Beldie, 1977; Ciocârlan, 2000, 2009) or Betulaceae family (Prodan, 1939; Georgescu, 1952; Prodan and Buia, 1966; Resmeriță, 1970). Corylus genus represents a diverse group of useful woody plants, ranging from small, multi-stemmed shrubs to tall, stately trees, all of which produce edible nuts (Molnar, 2011). Corylus genus is widely distributed across temperate regions of the Northern Hemisphere, with species found in Japan, Korea, and China, through Tibet, India, Northern Iran, Turkey, the Caucuses, Europe, and North America (Molnar, 2011). Corylus colurna L. (Turkish hazel or Turkish filbert) and C. avellana L. (Common hazel) are the most common hazel species in Europe (Riethmueller et al., 2014). C. avellana L., which has a wide distribution, while, C. colurna L., restricted to the Balkans, Romania, and Northern Turkey (Thompson et al., 1996). In spontaneous flora of Romania, Corylus genus is represented by 2 species: C. avellana L. and C. colurna L. In southern regions of Romania there is also C. maxima Mill. cultivated as well, a taxon synonymous with $C$. avellana, the difference between them being at the level of involucre (Mehlenbacher, 1991; Thompson et al., 1996; Erdogan and Mehlenbacher, 2000a, b). The spreading of $C$. colurna in Romania is quite small, only in Banat and Oltenia and Vâlcan Mountains is its northern limit of the area (Boruz et al., 2013). Corylus avellana is commonly found in both national and European levels. C. avellana is widely distributed in Europe, in natural stands ranging from 
Scandinavia to the south of the continent (Palmé and Vendramin, 2002). In the north it can be found in Norway up to $67^{\circ} \mathrm{N}$, although its northern limit decreases further to the east (Deacon, 1974). It is the first species to colonize the European continent since the last ice age, as evidenced by studies on pollen and chloroplast (Palmé and Vendramin, 2002; Boccacci and Botta, 2009). C. colurna is a calcicolous species that has its origin in the Balkans (Boruz et al., 2013) and is a member of the Europe-Siberia flora region (Molnar, 2011). C. avellana is the only cultivated species and contains a great amount of genetic diversity (Rovira, 1997). C. colurna is one of the common species in Turkey (Aydinoglu, 2010). Beside scientific information, also valuable information on the presence of certain species in certain ecological areas is obtained from herbarium collections. Herbal collections have a scientific, didactic, educational, and also informative value. The collection of plants began in the 16th century. Later, J.P. Tourefort (ca 1700, France) used the term "herbarium" for plants (Bridson and Forman, 1999). One of the oldest herbariums in South-Eastern Europe, a pre-Linnaean herbarium dating from 1734, is in the botanical collection of the Natural History Museum within Brukenthal Museum (Sibiu, Romania). Their purpose is important because they provide information about plants and their area of spread during long periods that help to carry out studies in taxonomy, biodiversity, ecology, anatomy, morphology etc. (Vechiu and Dinca, 2019). Evidence of the hazelnut species spread is the information presented in various herbariums. The aim of this article is to analyse the scientific information and the main herbariums regarding the presence of $C$. colurna species on the Romanian territory.

\section{Materials and Methods}

The material used to carry out this paper consisted in specialized sections that contained the species Corylus colurna in the floristic list or in the composition of some phytocenoses, according to the herbarium data from the main herbariums in the country (Bucharest, Cluj-Napoca, Iași and Craiova). The material is based also on the analysis of the data obtained following the trips made in different resorts where they are vegetating. The acronyms used for the herbariums consulted are: BUCA (Herbarium of Biology Institute of the Romanian Academy), CL (Herbarium of "Babeş-Bolyai" University Cluj-Napoca), I (Herbarium of “Al. I. Cuza" University Iași) and CRA (Herbarium of Craiova University). A data base for C. colurna was realized, focusing on the following data: plate number; Herbarium/Botanic Collection/Institution; Species name; Harvesting date; Harvesting place; the person who has collected the plant; conservation grade, coded with numbers from 1 to $4(1=$ well preserved plant, 2 = plant with parts detached from the plate, but still present, $3=$ plant detached from the plate, with missing parts and $4=$ plant detached and fragmented, with over $50 \%$ of its parts missing) (Vechiu and Dinca, 2019). For localities mapping was used the UTM Code, according to Lehrer and Lehrer (1990). The distribution maps of the species were obtained using Corolog 2010 Program, realized in Biology Institute of Bucharest. The program uses a data base access, with information from literature, herbarium and field and two types of maps, the maps of annual average temperature and precipitation in Romania. The Stereo 70 coordinates corresponding to the points where plant material was collected were inserted in the table.

\section{Results and Discussion}

C. colurna (Turkish hazel) is a less widespread tree species in Romania. Data on the species $C$. colurna in the Romanian flora are sporadic until the publication of the paper "Conspectul Florei Romaniei" (Grecescu, 1898) which speaks of $C$. colurna as a fairly common species in the Balkan Peninsula, which is found only in Mehedinti to Verciorova and Banat. Prodan (1939) in the paper "Flora for determining and describing the plants growing in Romania" states that there are 3 species of Corylus in the Romanian flora (C. avellana, $C$. colurna and $C$. maxima) and in $C$. maxima he gives $C$. tubulosa Willd. as synonymous. In Flora României 
(volume I, 1952, pp. 197-202), Georgescu (1952) mentions the same 3 species: C. avellana, C. colurna and C. maxima. In 2 of them he presents intraspecific variability. In C. colurna the variety glandulifera (C. Koch) A. DC is mentioned, and in C. avellana, the typica form C. K. Schn. Laubholzk. with the subforms: brachychlámys Spach and schizochlámys Spach. About C. maxima it is only stated that it is cultivated, but rarely. Prodan and Buia (1966) in "The small illustrated flora of Romania" in Corylusgenus they mention 3 species. In each species the main morphological characters, the flowering and fruiting period and the ecology are presented, and in $C$. avellana the cenotic affiliation is also given. Beldie (1977) also quotes 2 species ( $C$. avellana and C. colurna) in the paper "Flora of Romania - illustrated determinator of vascular plants" (pp. 79-80). Each key taxon has the main differentiating characters, lifespan, biological form, height, flowering period, spread over areas and floors of vegetation, resorts where they can be found, soil type, trophicity and geo-element. In "The illustrated flora of Romania Pteridophyta \& Spermatophyta" shows the presence of 3 species, 2 spontaneous (C. avellana and $C$. colurna) and one cultivated (C. maxima). Among the analysed plants by the authors new data on the chorology of their Corylus species were also found in various synthesis papers that had as object of study the flora and vegetation of a certain territory. Information on the spread of C. avellana: Pop (1968) in the Crisurilor Plain is frequently presented; Resmeriță (1970) in the Vlădeasa Massif; in the Eastern Carpathians (Oprea and Sîrbu, 2009); Rodna Mountains by Coldea (1990) etc. C. avellana species has a good representation in the literature. It was mentioned by Cârțu (1969) from in Amaradia river basin, by Cârțu (1972) in the area between Jiu-Desnățui-Craiova and the Danube, by Năstase (1982) in Bucovăț forest, by Karácsonyi (1995) in Satu-Mare county, by Oroian (1998) in Mureș Gorge, between Toplița and Deda, by Negrean et al. (2017) in Salaj county. These last authors also identified the $C$. colurna species in several points: Șimleul Silvaniei N, Măgura Șimleului, Băile Iaz. Data on the presence of $C$. colurna in Romania are rare. Near Oravița (Romania) exists a big stand ( $80 \mathrm{ha}$ ) of $C$. colurna, the maximum data of age was 329 years (Richter, 2016). C. colurna was identified in the Iron Gates Park (Mehedinți) and Cheile Nerei-Beușnița National Park (Caraș-Severin) by Borlea et al. (2006) in the forest of Domogled (Băile Herculane, Romania) by Manoiu et al. (2015) and Ștefan et al. (2013). C. colurna was also identified in arranged dendrological parks, parks that include a several plants, very rare, in Bistrița (Rusu and Gavriloaie, 2011), in "Bazoș Arboretum" protected area (Cadar et al., 2014), in Cheile Nerei-Beușnița Site (Biri et al., 2016). Turkish hazel is a tolerant semi-shade tree species that can create mixed stands with other species and has a low level of invasion potential (Šeho et al., 2019).

Table 1. Table 1 Corylus colurna inventory in Romania

\begin{tabular}{|c|c|c|c|c|c|c|c|c|}
\hline \multirow[b]{2}{*}{$\begin{array}{l}\text { Plate } \\
\text { no }\end{array}$} & \multirow{2}{*}{$\begin{array}{l}\text { Herbarium/ } \\
\text { Botanic } \\
\text { collection/ } \\
\text { Institution }\end{array}$} & \multirow[b]{2}{*}{$\begin{array}{c}\text { Harvesting } \\
\text { date }\end{array}$} & \multirow[b]{2}{*}{ Harvesting place } & \multirow[b]{2}{*}{$\begin{array}{c}\text { Collected/ } \\
\text { Determinated } \\
\text { by: }\end{array}$} & \multirow[b]{2}{*}{$\begin{array}{c}\text { Conservation } \\
\text { Degree }\end{array}$} & \multirow[b]{2}{*}{ COD_UTM } & \multicolumn{2}{|c|}{ Stereo70 } \\
\hline & & & & & & & $\mathrm{X}(\mathrm{N})$ & $\mathrm{Y}(\mathrm{E})$ \\
\hline 1 & I20946 & $\begin{array}{c}\text { August 23, } \\
1965\end{array}$ & $\begin{array}{c}\text { Dolj County, } \\
\text { Craiova town, } \\
\text { Parcul Poporului } \\
\text { - public park }\end{array}$ & $\begin{array}{l}\text { Leg. et det. } \\
\text { Nicolăescu. } \\
\text { Revised Țopa }\end{array}$ & 1 & GQ21 & 404569.4953 & 311780.9795 \\
\hline 1 & $\begin{array}{c}\text { I33672/ Flora } \\
\text { Romaniae } \\
\text { Exsiccata nr. } \\
392\end{array}$ & $\begin{array}{c}\text { May 27, } \\
1923\end{array}$ & $\begin{array}{c}\text { Caraş-Severin } \\
\text { County, } \\
\text { Domogled } \\
\text { Mountain, forest }\end{array}$ & $\begin{array}{l}\text { Leg. et det. } \\
\text { Borza \& } \\
\text { Nyarady }\end{array}$ & 1 & FQ28 & 297836.6474 & 377959.5701 \\
\hline 1 & I38321 & $\begin{array}{c}\text { July } 23 \text {, } \\
1972\end{array}$ & $\begin{array}{c}\text { Caraș-Severin } \\
\text { County, Băile } \\
\text { Herculane town, } \\
\text { Domogled } \\
\text { Mountain, } \\
\text { forested } \\
\text { limestone cliffs }\end{array}$ & $\begin{array}{l}\text { Leg. et det. } \\
\text { Sârbu }\end{array}$ & 1 & FQ18 & 296893.0355 & 378553.3915 \\
\hline 1 & $\begin{array}{c}\text { I44603 / Flora } \\
\text { Exsiccată } \\
\text { Forestieră a } \\
\text { R.P.R.nr. } 55\end{array}$ & $\begin{array}{l}\text { October } \\
12,1959\end{array}$ & $\begin{array}{c}\text { Caraș-Severin } \\
\text { County, Berzasca } \\
\text { village, on Sirina } \\
\text { river valley }\end{array}$ & $\begin{array}{l}\text { Leg. Grapini, } \\
\text { det. Beldie. }\end{array}$ & 1 & EQ74 & 258778.9364 & 355521.1017 \\
\hline
\end{tabular}


Radutoiu D and Cosmulescu S (2020). Not Bot Horti Agrobo 48(3):1709-1718

\begin{tabular}{|c|c|c|c|c|c|c|c|c|}
\hline 1 & $\begin{array}{l}\text { I44604 / Flora } \\
\text { Romaniae } \\
\text { Exsiccata nr. } \\
392 .\end{array}$ & $\begin{array}{l}\text { October 6, } \\
1922 \\
\text { May 27, } \\
1923\end{array}$ & $\begin{array}{l}\text { Caraș-Severin } \\
\text { County, Silvam } \\
\text { formans in } \\
\text { Domogled } \\
\text { Mountain, alt } \\
\text { cca } 700-900 \mathrm{~m}\end{array}$ & $\begin{array}{l}\text { Leg. Borza, } \\
\text { Borza \& } \\
\text { Nyarady }\end{array}$ & 1 & FQ18 & 296893.0355 & 378553.3915 \\
\hline 3 & $\begin{array}{c}\text { I44605, } \\
\text { I44606, I72701 } \\
\text { / Flora } \\
\text { Romaniae } \\
\text { Exsiccata nr. } \\
\text { 392b. } \\
\end{array}$ & $\begin{array}{c}\text { March 31, } \\
1945\end{array}$ & $\begin{array}{l}\text { Timis County, } \\
\text { Culta in horto } \\
\text { Muhle oppidi } \\
\text { Timisoara. Arbor } \\
12 \mathrm{~m} \text { alt. }\end{array}$ & $\begin{array}{c}\text { Leg. } \\
\text { Bujorean. } \\
\text { Revised Borza }\end{array}$ & 1 & ER16 & 206586.4622 & 478873.4057 \\
\hline 1 & $\begin{array}{c}\text { Herbarul } \\
\text { Grădinii } \\
\text { Botanice Iași / } \\
\text { Flora Romaniae } \\
\text { Exsiccata nr. } \\
\text { 392b. }\end{array}$ & $\begin{array}{c}\text { March 31, } \\
1945\end{array}$ & $\begin{array}{l}\text { Timis County, } \\
\text { Culta in horto } \\
\text { Muhle oppidi } \\
\text { Timisoara. Arbor } \\
12 \mathrm{~m} \text { alt. }\end{array}$ & $\begin{array}{c}\text { Leg. } \\
\text { Bujorean. } \\
\text { Revised Borza }\end{array}$ & 3 & ER16 & 206586.4622 & 478873.4057 \\
\hline 1 & $\begin{array}{l}\text { Herbarul } \\
\text { Grădinii } \\
\text { Botanice Iași }\end{array}$ & $\begin{array}{c}\text { June 21, } \\
1973\end{array}$ & $\begin{array}{c}\text { Caraș-Severin } \\
\text { County, Băile } \\
\text { Herculane town, } \\
\text { in Crucea Alba } \\
\text { forest }\end{array}$ & $\begin{array}{l}\text { Leg. et det. } \\
\text { Goga }\end{array}$ & 1 & FQ17 & 297012.6274 & 379327.0347 \\
\hline 2 & $\begin{array}{c}\text { Herbarul } \\
\text { Gradinii } \\
\text { Botanice Iași }\end{array}$ & $\begin{array}{c}\text { May 27, } \\
1951\end{array}$ & $\begin{array}{l}\text { Cluj County, } \\
\text { Borșa }\end{array}$ & $\begin{array}{l}\text { Leg. et det. } \\
\text { Țopa }\end{array}$ & 1 & GS09 & 397770.7925 & 604459.5031 \\
\hline 1 & $\begin{array}{c}\text { Herbarul } \\
\text { Gradinii } \\
\text { Botanice Iași }\end{array}$ & $\begin{array}{c}\text { June } 18, \\
1951\end{array}$ & & $\begin{array}{l}\text { Leg. et det. } \\
\text { Celan }\end{array}$ & 1 & & & \\
\hline 1 & BUCA150593 & $\begin{array}{c}\text { July 03, } \\
1931\end{array}$ & $\begin{array}{c}\text { Caraș-Severin } \\
\text { County, } \\
\text { Domogled } \\
\text { Mountain }\end{array}$ & $\begin{array}{l}\text { Leg. et det. } \\
\text { Grintescu }\end{array}$ & 1 & FQ28 & 297836.6474 & 377959.5701 \\
\hline 1 & BUCA131847 & $\begin{array}{c}\text { March 2, } \\
1966 \\
\end{array}$ & $\begin{array}{c}\text { Romania, district } \\
\text { Mehedinti }\end{array}$ & Leg. Dihoru & 1 & FQ16 & & \\
\hline 1 & BUCA131628 & $\begin{array}{c}\text { June 26, } \\
1964\end{array}$ & $\begin{array}{c}\text { Oltenia Region, } \\
\text { Tr. Severin } \\
\text { district, SE of } \\
\text { Ilovița forest, } \mathrm{N} \\
\text { Vârciorova } \\
\end{array}$ & $\begin{array}{l}\text { Leg. et det. } \\
\text { Roman }\end{array}$ & 1 & FQ15 & 300683.1994 & 363968.9998 \\
\hline 3 & $\begin{array}{c}\text { BUCA044836 } \\
\text { / Flora } \\
\text { Romaniae } \\
\text { Exsiccata nr. } \\
\text { 392b. }\end{array}$ & $\begin{array}{l}\text { March 31, } \\
1945\end{array}$ & $\begin{array}{l}\text { Timiș County, } \\
\text { Culta in horto } \\
\text { Muhle oppidi } \\
\text { Timisoara. Arbor } \\
12 \mathrm{~m} \text { alt. }\end{array}$ & $\begin{array}{c}\text { Leg. } \\
\text { Bujorean. } \\
\text { Revised Borza }\end{array}$ & 3 & ER16 & 206586.4622 & 478873.4057 \\
\hline 1 & $\begin{array}{l}\text { BUCA/Flora } \\
\text { Romaniae } \\
\text { Exsiccata nr. } \\
392\end{array}$ & $\begin{array}{c}\text { May 27, } \\
1923\end{array}$ & $\begin{array}{c}\text { Caraș-Severin } \\
\text { County, } \\
\text { Domogled } \\
\text { Mountain, forest }\end{array}$ & $\begin{array}{l}\text { Leg. et det. } \\
\text { Borza \& } \\
\text { Nyarady }\end{array}$ & 1 & FQ28 & 297836.6474 & 377959.5701 \\
\hline 1 & BUCA & $\begin{array}{l}\text { March 26, } \\
1948\end{array}$ & $\begin{array}{l}\text { Mehedinti } \\
\text { District, in a } \\
\text { forest on } \\
\text { Marcopriciu, } \\
\text { towards } \\
\text { Varciorova }\end{array}$ & Leg. Ularu & 1 & FQ15 & 312713.0338 & 391274.319 \\
\hline 1 & $\begin{array}{c}\text { BUCA / Flora } \\
\text { Exsiccata } \\
\text { Forestiera a } \\
\text { R.P.R. nr. 55 }\end{array}$ & $\begin{array}{l}\text { October } \\
12,1959\end{array}$ & $\begin{array}{c}\text { Caraș-Severin } \\
\text { County, } \\
\text { Berzasca, on } \\
\text { Sirina river valley }\end{array}$ & $\begin{array}{l}\text { Leg. Grapini, } \\
\text { det. Beldie. }\end{array}$ & 1 & EQ74 & 258778.9364 & 355521.1017 \\
\hline 1 & BUCA011749 & $\begin{array}{l}\text { September } \\
09,1949\end{array}$ & $\begin{array}{c}\text { Hunedoara } \\
\text { Region, Simeria } \\
\text { Dendrological } \\
\text { Park } \\
\end{array}$ & & 1 & FR57 & 346196.3421 & 486659.6678 \\
\hline 2 & CL661848 & $\begin{array}{c}\text { July } 28, \\
2003\end{array}$ & $\begin{array}{l}\text { Romania, Banat } \\
\text { region, } \\
\text { Mehedinți }\end{array}$ & $\begin{array}{l}\text { Leg. et det. } \\
\text { Negrean }\end{array}$ & 1 & FQ04 & 282570.2909 & 348429.2714 \\
\hline
\end{tabular}


Radutoiu D and Cosmulescu S (2020). Not Bot Horti Agrobo 48(3):1709-1718

\begin{tabular}{|c|c|c|c|c|c|c|c|c|}
\hline & & & $\begin{array}{c}\text { District, } \\
\text { Cazanele Mari, } \\
\text { Ciucarul Mare } \\
\text { Mount Peak }\end{array}$ & & & & & \\
\hline & CL642553 & $\begin{array}{c}\text { May 25, } \\
1988\end{array}$ & $\begin{array}{c}\text { Banat Reion, } \\
\text { Caraș-Severin } \\
\text { district, } \\
\text { Domogled Mt., } \\
\text { in the forest } \\
\text { above Băile } \\
\text { Herculane town }\end{array}$ & $\begin{array}{l}\text { Leg. et det. } \\
\text { Groza }\end{array}$ & 1 & FQ17 & 296345.3594 & 380056.3988 \\
\hline 1 & CL389671 & $\begin{array}{l}\text { August 29, } \\
1948\end{array}$ & $\begin{array}{l}\text { Oltenia region, } \\
\text { Gorj district, } \\
\text { Chestnut forest } \\
\text { in Tismana } \\
\text { village }\end{array}$ & Leg. Borza & 1 & FQ59 & 337669.2452 & 400170.29 \\
\hline 1 & CL617491 & $\begin{array}{c}\text { May 20, } \\
1939\end{array}$ & Cazane & $\begin{array}{l}\text { Leg. Pop, det. } \\
\text { Schneider }\end{array}$ & 1 & FQ04 & 284783.8028 & 352200.8366 \\
\hline 1 & $\begin{array}{l}\text { CL558359 / } \\
\text { Flora Exsiccata } \\
\text { Forestiera a } \\
\text { R.P.R. nr. 55 } \\
\end{array}$ & $\begin{array}{l}\text { October } \\
12,1959\end{array}$ & $\begin{array}{c}\text { Caraș-Severin } \\
\text { County, } \\
\text { Berzasca, on } \\
\text { Sirina river valley }\end{array}$ & $\begin{array}{l}\text { Leg. Grapini, } \\
\text { det. Beldie }\end{array}$ & 1 & EQ74 & 258778.9364 & 355521.1017 \\
\hline 1 & CL649436 & $\begin{array}{c}\text { August 28, } \\
1983\end{array}$ & $\begin{array}{c}\text { Domogled } \\
\text { Mountain, above } \\
\text { Băile Herculane } \\
\text { town }\end{array}$ & Leg. Boscaiu & 1 & FQ17 & 296345.3594 & 380056.3988 \\
\hline 1 & CL617403 & $\begin{array}{c}\text { May 19, } \\
1939 .\end{array}$ & $\begin{array}{c}\text { Above Băile } \\
\text { Herculane town }\end{array}$ & $\begin{array}{l}\text { Leg. Pop, det. } \\
\text { Schneider }\end{array}$ & 1 & FQ17 & 296345.3594 & 380056.3988 \\
\hline 9 & $\begin{array}{l}\text { CL80911, } \\
\text { CL82357, } \\
\text { CL95249, } \\
\text { CL95250, } \\
\text { CL95257, } \\
\text { CL95258, } \\
\text { CL95268, } \\
\text { CL95266, } \\
\text { CL95267 }\end{array}$ & $\begin{array}{c}\text { May 20, } \\
1920\end{array}$ & $\begin{array}{l}\text { Caraș-Severin } \\
\text { County, Băile } \\
\text { Herculane town, } \\
\text { limestone cliffs } \\
\text { on Suscului Peak }\end{array}$ & Leg. Borza & 1 & FQ17 & 296345.3528 & 380056.3952 \\
\hline 2 & $\begin{array}{l}\text { CL215680, } \\
\text { CL215681 }\end{array}$ & $\begin{array}{c}\text { June } 29 \\
1948 .\end{array}$ & $\begin{array}{c}\text { Banat region, } \\
\text { Caraș district, on } \\
\text { Beul sec river } \\
\text { valley }\end{array}$ & $\begin{array}{c}\text { Leg. Borza et } \\
\text { Buia }\end{array}$ & 1 & FQ06 & 246161.9903 & 385618.3098 \\
\hline 3 & $\begin{array}{l}\text { CL55918, } \\
\text { CL207860, } \\
\text { CL617404 }\end{array}$ & $\begin{array}{l}\text { March 31, } \\
1945\end{array}$ & $\begin{array}{l}\text { Timis County, } \\
\text { Culta in horto } \\
\text { Muhle oppidi } \\
\text { Timișoara. Arbor } \\
12 \mathrm{~m} \text { alt. }\end{array}$ & $\begin{array}{c}\text { Leg. } \\
\text { Bujorean. } \\
\text { Revised Borza }\end{array}$ & 2 & ER16 & 206586.4622 & 478873.4057 \\
\hline 1 & CL589654 & $\begin{array}{c}\text { August 23, } \\
1942\end{array}$ & $\begin{array}{c}\text { Banat region, } \\
\text { Caraș district, on } \\
\text { Beul sec river } \\
\text { valley }\end{array}$ & $\begin{array}{l}\text { Leg. Borza et } \\
\text { Ogruțan }\end{array}$ & 1 & FQ06 & 246161.9903 & 385618.3098 \\
\hline 1 & CL215200 & $\begin{array}{l}\text { September } \\
09,1949\end{array}$ & $\begin{array}{c}\text { Hunedoara } \\
\text { region, Simeria } \\
\text { Dendrological } \\
\text { Park }\end{array}$ & & 1 & FR57 & 346196.3421 & 486659.6678 \\
\hline 1 & CL591886 & $\begin{array}{c}\text { May 19, } \\
1939\end{array}$ & $\begin{array}{l}\text { Banat region, } \\
\text { Severin district, } \\
\text { above Băile } \\
\text { Herculane town }\end{array}$ & $\begin{array}{c}\text { Leg. Borza et } \\
\text { Todor }\end{array}$ & 1 & FQ17 & 296345.3594 & 380056.3988 \\
\hline 1 & $\begin{array}{c}\text { CL133814 / } \\
\text { Flora Romaniae } \\
\text { Exsiccata nr. } \\
392 \\
\end{array}$ & $\begin{array}{c}\text { May 27, } \\
1923\end{array}$ & $\begin{array}{c}\text { Caraș-Severin } \\
\text { County, } \\
\text { Domogled } \\
\text { Mountain, forest }\end{array}$ & $\begin{array}{l}\text { Leg. et det. } \\
\text { Borza \& } \\
\text { Nyarady }\end{array}$ & 1 & FQ28 & 297836.6474 & 377959.5701 \\
\hline 2 & $\begin{array}{c}\text { CL21506, } \\
21509\end{array}$ & & $\begin{array}{c}\text { Cerna river } \\
\text { Valley }\end{array}$ & Leg. Pavai & 1 & FQ39 & 302949.8893 & 391210.8189 \\
\hline
\end{tabular}


Radutoiu D and Cosmulescu S (2020). Not Bot Horti Agrobo 48(3):1709-1718

\begin{tabular}{|c|c|c|c|c|c|c|c|c|}
\hline 1 & $\begin{array}{c}\text { CRA / Flora } \\
\text { Romaniae } \\
\text { Exsiccata nr. } \\
392\end{array}$ & $\begin{array}{c}\text { May 27, } \\
1923\end{array}$ & $\begin{array}{c}\text { Caras-Severin } \\
\text { County, } \\
\text { Domogled } \\
\text { Mountain, forest }\end{array}$ & $\begin{array}{c}\text { Leg. et det. } \\
\text { Borza \& } \\
\text { Nyarady }\end{array}$ & 1 & FQ28 & 297836.6474 & 377959.5701 \\
\hline 1 & $\begin{array}{c}\text { CRA / Flora } \\
\text { Exsiccata } \\
\text { Forestiera a } \\
\text { R.P.R. nr. 55 }\end{array}$ & $\begin{array}{c}\text { October } \\
12,1959\end{array}$ & $\begin{array}{c}\text { Caraș-Severin } \\
\text { County, Berzasca } \\
\text { village, on Sirina } \\
\text { river valley }\end{array}$ & $\begin{array}{c}\text { Leg. Grapini, } \\
\text { det. Beldie. }\end{array}$ & 1 & EQ74 & 258778.9364 & 355521.1017 \\
\hline 1 & CRA18152 & $\begin{array}{c}\text { April 17, } \\
2004\end{array}$ & $\begin{array}{c}\text { Mehedinți } \\
\text { County, } \\
\text { Comănești Hills }\end{array}$ & $\begin{array}{c}\text { Leg. I. } \\
\text { Costache }\end{array}$ & 1 & FQ47 & 331161.1437 & 385725.9954 \\
\hline
\end{tabular}

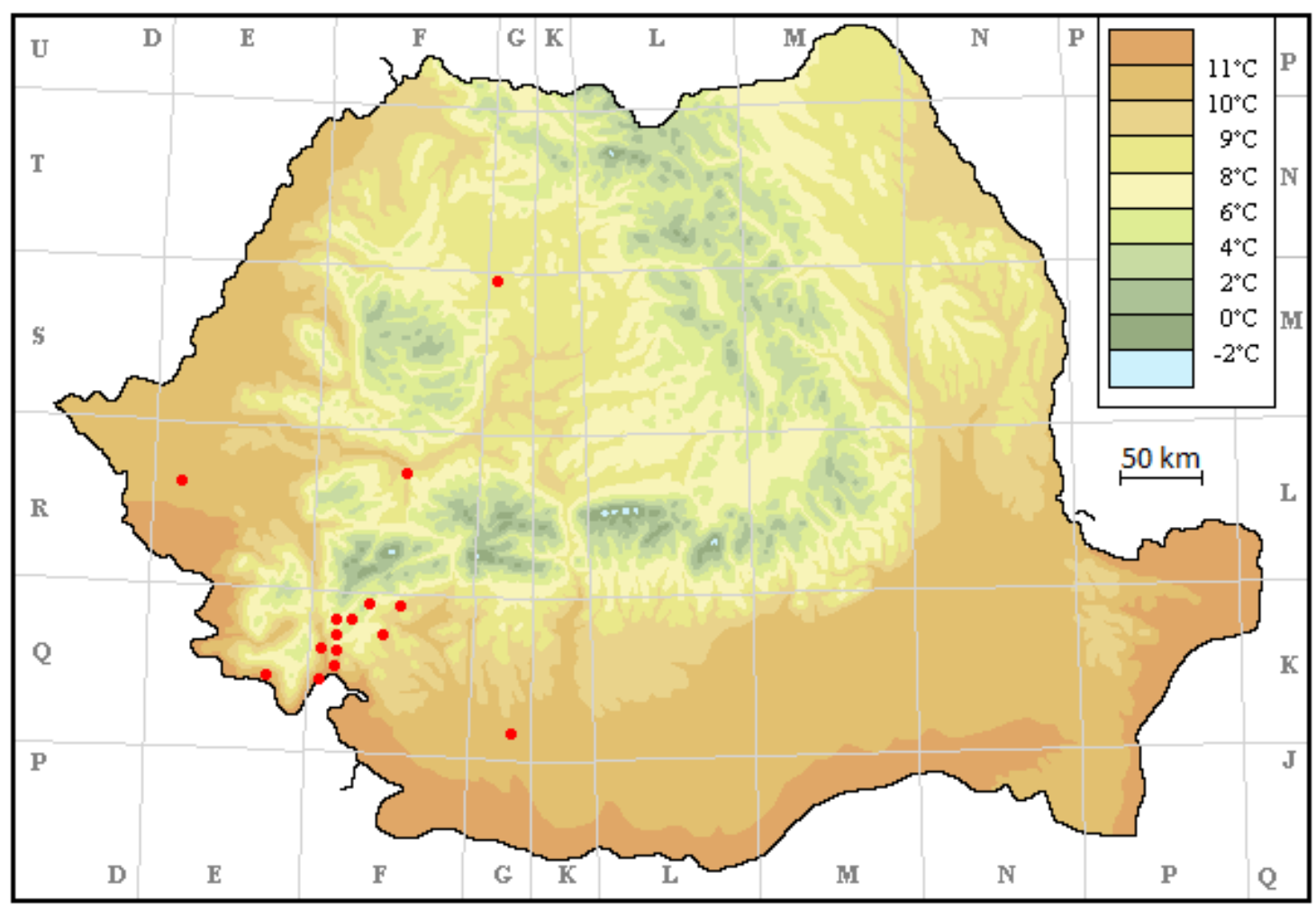

Figure 1. Distribution of species C. colurna correlated with annual average temperature

Herbarium data with this taxon are also scarce (Table 1). Herbarium material from Iaşi (I), Cluj (CL), Bucharest (BUCA) and Craiova (CRA) shows that the oldest plant, belonging to C. colurna species, was collected in 1920 from Caraș-Severin County, Băile Herculane (calcareous rocks on Suscului peak) by Al. Borza (Figure 1). Also, during period 1920-1930, information appears regarding the identification of some C. colurna specimens on the territory of Caraș-Severin county, evidence introduced in the herbariums in Iași and Bucharest. Most plants were harvested during 1940-1949 and are preserved in the herbariums of Bucharest, Cluj and Iași. The last plant preserved in Craiova herbarium was harvested in 2004, from Mehedinți area, Dealul Comăneștilor, by Iulian Costache. The place of sampling is located in Romania (Figures 1 and 2). The plants were collected mainly from the southwestern part of Romania. The county from which most specimens were collected is Caraș-Severin, especially in the areas of Mount Domogled, above Băile Herculane; Mehedinți - Cazanele Mari, Ciucarul Mare peak, Comăneștilor Hill, in the forest on Marcopriciu, towards Vârciorova; Hunedoara - Simeria Dendrological Park, Gorj - Tismana, Dolj - Craiova. The analysis of the data shows that the distribution of $C$. colurna in Romania is quite small, only in Banat and Oltenia (ie southern Banat, Mehedinți Mountains, Mehedinți Plateau and Motru Hills) (Boruz et al., 2013) and is found in different parks, 
in areas favourable for the cultivation of this species. The presence of $C$. colurna species is also indicated by Bușe Dragomir and Nicolae (2014) in the Iron Gates park, Mehedinți. Turkish hazel has been found sporadically in forest habitats in Central, Eastern and Western Serbia (Mitrovic et al., 2001). Therefore, the remaining Turkish hazel populations in different locations are of critical importance, and the genetic conservation of this species plays a key role in the sustainable development of forests. Corylus species have scientific and practical importance. C. colurna has no relevant economic importance, but agronomic characteristics are very important, it does not produce basal shoots, an important aspect for hazelnut cultivation (Avanzato, 2008). Biodiversity conservation strategy should include forest protected areas as important objectives (Borlea et al., 2006). The people who collected and identified the plants are Romanian specialists, famous botanists: A. Borza, E.J. Nyárády, A. Nicolaescu, E. Topa, I. Sârbu, V. Grapini, A. Beldie, G. Bujorean, I. Goga, E. T, sopa, M. Celan, G. Grintescu, G. Dihoru, N. Roman, G. Bujorean, I. Ularu, G. Negrean, Gh. Groza, E. Pop, E. Schneider, Monica Boșcaiu, E. Pop, Al. Buia, G. Bujorean, P. Ogruțan, I. Todor, Hb. Pavai, I. Costache.

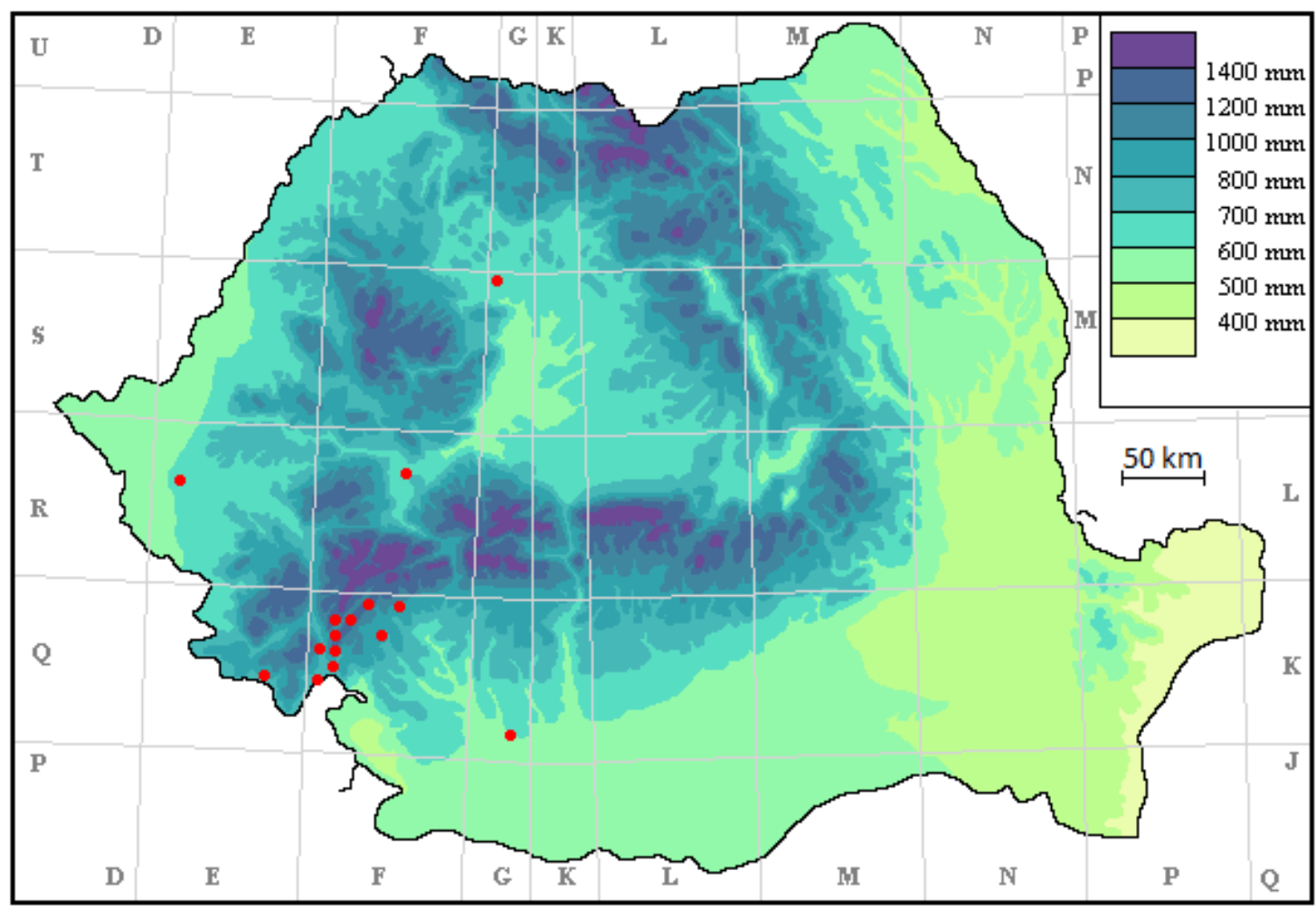

Figure 2. Distribution of species $C$. colurna correlated with annual average precipitation

\section{Conclusions}

In conclusion, the presence of $C$. colurna species on the Romanian territory is signalled about 122 years ago in the paper "Conspectus of Romanian Flora". Subsequent scientific information identifies the species in different locations, especially in the South-Western Romania, in dendrological and natural parks. Scientific importance of $C$. colurna species lies in its rarity, the species has an area limit on the territory of Romania and in the value, it gives to the natural ecosystems in which it grows. The practical value is given by the edible fruits, 
valuable from a food or medicinal point of view and by its agronomic characteristics (it does not produce basal shoots).

\section{Authors' Contributions}

Conceptualization of research (SC, DR); Design of the experiments (SC); Contribution of experimental materials (DR); Analysis of data and interpretation (SC, DR); Preparation of the manuscript (SC, DR). Both authors read and approved the final manuscript.

\section{Acknowledgements}

Thanks to our colleagues who have provided chorology information from different collections: M. Puşcaş (Al. Borza Botanical Garden, Cluj-Napoca), A. Oprea (A. Fătu Botanical Garden, Iaşi), Irina Blaj (Curator of Herbarium, Alexandru Ioan Cuza University Iași), S. Ştefănuț (Romanian Academy, Biology Institute).

\section{Conflict of Interests}

The authors declare that there are no conflicts of interest related to this article.

\section{References}

Avanzato D (2008). Farming affects plant genetic erosion: policy actions to prevent it. Scientific Papers of the Research Institute for Fruit Growing Pitești, Romania XXIV:9-14. http://agris.fao.org/agrissearch/search.do?recordID $=$ DJ2012060153

Aydinoglu AC (2010). Examining environmental condition on the growth areas of Turkish hazelnut (Corylus colurna L.). African Journal of Biotechnology 9:6492-6502. http://Www.academicjournals.org/AJB

Beldie Al (1977). Flora României. Determinator ilustrat al plantelor vasculare [Flora of Romania. Illustrated determinator of vascular plants]. Editura Academiei Române, Bucuresti.

Biri IA, Teodosiu M, Turcu DO, Merce O, Loren A, Apostol J, Marcu C (2016). 24000 ha of primary beech forests, the Romanian proposal in UNESCO World Heritage. Bucovina Forestieră 16:107-112.

Boccacci P, Botta R (2009). Investigating the origin of hazelnut (Corylus avellana L.) cultivars using chloroplast microsatellites. Genetic Resources and Crop Evolution 56:851-859. https://doi.org/10.1007/s10722-0099406-6

Borlea GF, Radu S, Stana D (2006). Forest biodiversity preservation in Romania. Notulae Botanicae Horti Agrobotanici Cluj-Napoca 34:21-26. https://doi.org/10.15835/nbha341258

Boruz V, Dihoru G, Răduțoiu D (2013). Areal limit in the Romanian territory: 7. Corylus colurna. Journal of Horticulture, Forestry and Biotechnology 17(4):5-9.

Bridson D, Forman L (1999). The herbarium handbook. Royal Botanic Gardens. Kew, Richmond, Surrey, United Kingdom.

Bușe Dragomir L, Nicolae I (2014). Ecophysiological aspects regarding the, sibleac, associations from Iron Gates Natural Park. Annals of the University of Craiova Agriculture, Montanology, Cadastre Series 44(1):33-38.

Cadar N, Merce O, Turcu DO, Cântar IC (2014). Management measures in Bazos Arboretum protected area. Journal of Horticulture, Forestry and Biotechnology 18(3):30-33.

Cârțu D (1972). Vegetația lemnoasă dintre Jiu-Desnățui-Craiova și Dunăre [The woody vegetation in the area between Jiu-Desnățui-Craiova and the Danube]. Studii și Cercetări 213-222. 
Cârțu M (1969). Materiale pentru flora și vegetația Bazinului hidrografic al Amaradiei [Materials for the flora and vegetation of Amaradia River Basin]. Annals of the University of Craiova I(XI): 37-44.

Ciocârlan V (2000). Flora ilustrată a României. Pteridophyta et Spermatophyta [The illustrated flora of Romania. Pteridophyta et Spermatophyta]. Editura Ceres, Bucureşti.

Ciocârlan V (2009). Flora ilustrată a României. Pteridophyta et Spermatophyta [The illustrated flora of Romania. Pteridophyta et Spermatophyta]. Editura Ceres, Bucureşti.

Coldea G (1990). Munții Rodnei. Studiu geobotanic [Rodna Mountains. Geobotanical study]. Editura Academiei Române, Bucureşti.

Deacon J (1974). The location of refugia of Corylus avellana L. during the Weichselian glaciation. New Phytologist 73:1055-1063. https://doi.org/10.1111/j.1469-8137.1974.tb01331.x

Dihoru G (1975). Învelişul vegetal din muntele Siriu [Vegetal cover of Siriu mountain]. Editura Academiei Române Bucureşti.

Erdogan V, Mehlenbacher SA (2000a). Interspecific hybridization in hazelnut (Corylus). Journal of the American Society for Horticultural Science 125:489-497. https://doi.org/10.21273/JASHS.125.4.489

Erdogan V, Mehlenbacher SA (2000b). Phylogenetic relationships of Corylus species (Betulaceae) based on nuclear ribosomal DNA ITS region and chloroplast matK gene sequences. Systematic Botany 25:727-737. https://doi.org/10.2307/2666730

Georgescu CC (1952). Betulaceae. In Săvulescu \& al. Flora R.P.R. Edit. Acad. Române. Bucureşti pp 189-217.

Grecescu D (1898). Conspectul florei României: Plantele vasculare indigene și cele naturalizate ce se găsesc pe teritoriul României, considerate subt punctul de vedere sistematic și geographic [The Romanian flora perspective: Indigenous and naturalized vascular plants found on the Romanian territory, considered from a systematic and geographical point of view]. Tipografia Dreptatea, București.

Karácsonyi C (1995). Flora și vegetația județului Satu Mare [Flora and vegetation of Satu Mare county]. Editura Muzeului Sătmărean. Satu Mare.

Lehrer A, Lehrer M (1990). Cartografierea faunei şi florei României (Coordonate arealografice) [Mapping of the Romanian fauna and flora (Area coordinates)]. Editura Ceres. Bucureşti

Mănoiu VM, Crăciun AI, Spiridon RM (2015). The geoecological structure typical for the depression basin of the Băile Herculane resort, Romania. Proceedings of ADVED15 International Conference on Advances in Education and Social Sciences, Istanbul, Turkey pp 116-126.

Mehlenbacher SA (1991). Hazelnuts (Corylus). Acta Horticulturae 290:791-838. https://doi.org/10.17660/ActaHortic.1991.290.18

Mitrovic M, Stanisavljevic M, Ogasanovic D (2001). Turkish tree hazel biotypes in Serbia. Acta Horticulturae 556:191196. https://doi.org/10.17660/ActaHortic.2001.556.27

Molnar TJ (2011). Corylus. In: Wild Crop Relatives: Genomic and Breeding Resources. Springer, Berlin, Heidelberg pp $15-48$.

Năstase A (1982). Catalogul colecției botanice a muzeului Olteniei din Craiova, județul Dolj. (I). [Botanical collection catalogue of Oltenia Museum in Craiova, Dolj County]. Oltenia Studii și Comunicări Istorie, Etnografie, Științele Naturii pp 229-264.

Negrean G, Karácsonyi C, Szatmari PM (2017). Patrimoniul natural al Sălajului. Vol. I. Flora, microbiota și vegetația [The Natural Heritage of Sălaj county. Vol. I. Flora, microbiota and vegetation]. Editura Someșul, Satu Mare.

Oprea A, Sîrbu C (2009). Diversitatea floristică a munților Stânișoarei. (Carpații Orientali) [Floristic diversity of Stânişoara mountains. (Eastern Carpathians)]. Editura Universităţii A. I. Cuza, Iaşi.

Oroian S (1998). Flora şi vegetaţia defileului Mureşului între Topolniţa şi Deda [Flora and vegetation of Mureş River Gorge between Topolnița and Deda]. Casa de Editură Mureş. Tg. Mureş.

Palmé AE, Vendramin GG (2002). Chloroplast DNA variation, postglacial recolonization and hybridization in hazel, Corylus avellana. Molecular Ecology 11(9):1769-1779. https://doi.org/10.1046/j.1365-294X.2002.01581.X

Pop I (1968). Flora şi vegetaţia Câmpiei Crişurilor. Interfluviul Crişul Negru-Crişul Repede [Flora and vegetation of Crişurilor Plain. Crişul Negru-Crişul Repede Inter-rivers]. Editura Academiei Române, Bucureşti.

Prodan I (1939). Flora pentru determinarea şi descrierea plantelor ce cresc în România [Flora for determination and description of plants growing in România]. Tipografia Cartea Românească Cluj.

Prodan I, Buia Al (1966). Flora mică ilustrată a României (ed V) [The illustrated small flora of Romania (5th ed)]. Edit. Agro-Silvică, București. 
Resmeriță I (1970). Flora, vegetația și potențialul productiv pe masivul Vlădeasa [Flora, vegetation and productive potential on Vlădeasa massif]. Editura Academiei Române.

Richter E (2016). Corylus colurna stand near Oravita, Romania. Revista Pădurilor 131:19-26.

Riethmueller E, Toth G, Albert, A, Sonati M, Kery A (2014). Antioxidant activity and phenolic composition of Corylus colurna. Natural Product Communications 9:679-682.

Rovira M (1997). Genetic variability among hazelnut (C. avellane L.) cultivars. Acta Horticulturae 445:45-50. https://doi.org/10.17660/ActaHortic.1997.445.6

Rusu C, Gavriloaie C (2011). Unele considerații cu privire la vegetația ariilor protejate din municipiul Bistrița (BistrițaNăsăud, România) [Some considerations regarding the vegetation of protected areas in Bistrița municipality (Bistrița-Năsăud, Romania)]. Ecoterra 27:45-48.

Šeho M, Ayan S, Huber G, Kahveci G (2019). A review on Turkish hazel (Corylus colurna L.): a promising tree species for future assisted migration attempts. South-east European Forestry 10:53-63. https://doi.org/10.15177/seefor.19-04

Ștefan C, Chisaliță I, Moatar M, Stanciu SM, Fora CG, Banu C, Olaru D (2013). Future plans for developing the forestry and countryside and in the Natural Reserve Domogled. Journal of Horticulture, Forestry and Biotechnology 17:324-328.

Thompson MM, Lagerstedt HB, Mehlenbacher SA (1996). Hazelnuts. In: Janick J, Moore JN (Eds) Fruit breeding: nuts. Wiley, New York pp 125-184.

Vechiu E, Dinca L (2019). Characterization of Cornus plant present in Al. Beldie herbarium. Research Journal of Agricultural Science 51:169-175.
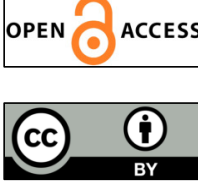

The journal offers free, immediate, and unrestricted access to peer-reviewed research and scholarly work. Users are allowed to read, download, copy, distribute, print, search, or link to the full texts of the articles, or use them for any other lawful purpose, without asking prior permission from the publisher or the author.

License - Articles published in Notulae Botanicae Horti Agrobotanici Cluj-Napoca are Open-Access, distributed under the terms and conditions of the Creative Commons Attribution (CC BY 4.0) License. (c) Articles by the authors; UASVM, Cluj-Napoca, Romania. The journal allows the author(s) to hold the copyright/to retain publishing rights without restriction. 